starration, (from excess of vigour,) and languid sloughings from its consequence; nercury, by violent inflammation, and rapid phagedenic ulcerations. Similar in the direction of their powers, but dissinilar in their modus operandi of effecting the same ends, they are very valuable agents in the treatment of particular diseases. In some, iodine, per se, may be preferred to mercury; in others, mercury to iodine; but in my limited experience, $I$ have found that they are more powerful when in combination. They mutually assist each other, their characteristic influences dereloping themselves as one or the other, is the dominant agent.

Nor does it seem absolutely requisite that iodine should be administered internally, seeing that its characteristic effects can be produced with equal cer. tainty and rapidity when applied to the skin, as in $\mathrm{Mr}$. Ray's striking case of hydrocephalus, and the interesting example of ganglion, brought forward by Dr. Toogood. To these, and in support of the principle which they. involve, I am happy to add the following cases from my own practice, wherein the external application of the undiluted tincture of iodine produced speedy and effectual cures :-

Conjoined with the unguentum hydrargyri nitratis in tinea cupitis, after the first inflammatory action was subdued.

In ulcerated and sloughing tonsils, both from common and specific inflammation.

Very much diluted in cynanche, after the capsicum and alum gargle had been used; its power was very marked.

In lepra, after the application of nitric acid.

In that disease of the knee-joint, called housemaid's knee, applying the tincture after cupping and blistering seems very efficucious.

- In acne, after the application of the lancet.

In bydrocele, (now under treatiment,) the water is being absorbed very rapidly from painting the scrotum with the tincture, and taking five grains' of blue pill twice a day.

My last instance is one which will startle your professional readers, for it is a case of polypus of the nose that has very nearly disappeared under the external use of the tincture. I discovered that the disease existed about a year since; an operation was advised, the tumour being moveable, and very low in the left nostril; the patient being timid, refused. I then commenced applying iodine, and at this moment no visible traces of the polypus remain; although from the passage offering obstruction to free nasal respiration, its roots are not destroyed.

I will here conclude the subject, with the hope that other of our members will bring forward the results of their experience, in the administration and application of this interesting remedial agent.

I have the honour to be, Sir,

Your obedient Servant,

CONWAY T. EDWARDS.

Bath Easton, Bath, January 7, 1846.

\section{UNIVERSITY COLLEGE HOSPITAL.}

\section{CASE CF PARALYSIS OP THE PORTIO-DURA NERVE.}

(Reported by Mr. John ElLlotT Wood.)

James Sherring, aged 20, single, admitted June 13th, 1845, under the care of Dr. A. T. Thompson. He is of moderate stature and conformation, light complexion, and nervo-sanguine temperament. His habits have always been regular, and he has taken very little stimulating liquor. He has had plenty of food and clothing, and a fair time allowed for sleep. By trade he is a painter, and he has followed this occupation for six years without interruption. He has lived in London for twelve years, in an open and airy situation. His father died of phthisis at the age of twenty-two; his mother is still living; and has always enjoyed good health. His own state of health has always been good, but he is now much thinner than formerly, though by no means emaciated. His state of mind is cheerful. The present attack commenced five days ago, (June 8th ;) previous to this, however, be had a slight cold in his head, with ringing in the ears, and pulsation in the temples. On the morning of the 8th June, on getting up, he found that there was paralysis of the right side of the face; he could not articulate clearly, nor close the eye on the affected side. This state continued till his admission into the Hospital on June 13th.

The following description will convey an accurate idea of the patient's appearance at this time. "When the portio dura is affected, the general sensibility remains intact, while the muscles of the face to which its branches are distributed are paralysed. The aspect of the face differs according as the muscles are in a state of repose or activity. In the former case all expression is lost in the paralysed part; the two sides of the face are not symmetrical, and when viewed by themselres apparently belong to different individuals. The features generally are dragged to the sound side; the labial conmissure of the paralysed part is drawu rather downwarks, and is brought nearer the median line; the mouth is oblique, and its centre does not correspond to the axis of the body. The paralysed half of the face is a. little more prominent than the sound one, which is wrinkled, contracted, and concealed behind the other when viewed in profile. The paralysed part appears broader than the sound one, while the eyelids are opened wide, and the eye appears more voluminous than its fellow. When on the other. hand the individual speaks, laughs, cries, sneezes, or coughs, the deformity of the countenance is much increased, the mouth and features remaining perfectly motionness on the paralysed side, while on the other they appear thrown into inordinate action. Mastication, however, is readily performed, and the patient can hold solid bodies between the teeth."*

Sensibility did not seem to be at all impaired on the affected side; the temperature was the same on both sides; there was no headache, nor tinnitus aurium; the appetite was good, the bowels regular, and every function appeared to be naturally performed. No tumour, nor enlarged gland, could be discovered in the 
neighbourhood of the parotid ; the patient was not, at this time, suffering from catarrh; there was no tenderness on pressure in the course of the nerve, but the hearing was decidedly impaired on the paralysed side. The pupils were equally dilated. He had never had the painter's colic, nor any severe fllness whatever. Previous to his admission he had been bled in the arm.

June 14th. A blister to be applied under the right ear, and the surface to be dressed with a sixth of ? grain of strychnia night and morning. Five grains of mercury with chalk to be taken every eight hours.

17th. He can hear a little better, and open the mouth with greater ease. The strychnia has not produced any twitchings. To be increased to a fifth of a grain.

19th. Increase the strychnia to a quarter of a grain night and morning.

21st. There is no improvement; he cannot close the eye any better, though one of the patients states that during sleep it is closed naturally; the blistered surface is sore to the touch, though the strychnia has produced no effect. Cupping under the right ear to twelve ounces.

24th. He thought himself a little better after the cupping; but the improvement, if any, is very slight.

26th. Cupping to eight ounces from the same place. The gums are affected by the mercury.

28th. A grain of strychnia to be applied to the blistered surface night and morning. It was ascertained, by testing with nitric acid, that the alkaloid was perfectly pure, and contained no brucia.

July 1st. There has been no twitching whatever, though the application of the strychnia causes pain and smarting. There is no amelioration. He feels weak, but in other respects perfectly well; there are no head symptoms; the appetite is good, and the bowels regular.

In this state he remained till July 22nd, when galvanism was applied along the course of the nerve. The mercury, which had produced slight salivation, had been omitted, and full diet with a tonic administered. After the use of galvanism daily for some days, he began to show decided symptoms of amelioration, and on the 26th he could close the eye considerably better, and the expression of the face : had decidedly improved. Articulation was more distinct, and he felt better in health and spirits. Galvanism was persevered in till his discharge, on August 16th, and thongh the good effects of the remedy were not so particularily striking afterwards as they were on its first application, yet sufficient improvement took place to show its beneficial influence very unequirocally. When discharged, he could very nearly close the right eye, and his countenance had lost that hideous expression which it had on his admission, though it was by no means symmetrical. A little deafnèss was still remaining.

The causes of the paralysis in this case conld never be clearly astertained. The deafness would have led one to suspect disease of the nerve before its exit from the cranium; but the sudden invasion of the disease, and the total absence of all symptoms referrible to the brain, would negative this supposition. There were no symptoms indicating the presence of lead in the system, a cause assigned by some uuthors as producing this affection; nor could any mechanical cause, as the pressure of a tumour, or of a spiculum of bone; be discovered after the most careful examination.

A remarkable circumstance in the case, is the total failure of the powerful agent strychnia in producing its ordinary effects, though the general senvibility of the part did not appear to be impaired. The very large quantity of a grain and a quarter applied to the blistered surface night and morning produced no inconvenience. Dr. J. L. Bardsley, of Manchester, strongly recommends the employment of strychnia in fucial paralysis, and enumerates twenty-three case in which it was beneficial. . He has also seen galvanism advantageously employed. It is more than probable that the disease would increase in severity after the patient's dismissal from the Hospital.

\section{PROVINCIAL \\ fltedical \& Eurgical Journal.}

\author{
WEDNESDAY, JANUARY 21, 1846.
}

In the Journal of last week were some remarks by a correspondent on the employment of incompetent persons in the dispensing of medicines. We have recently had an instance of the mischiefs resulting from such a practice, through a mistake; in the reading of a prescription, which no person competent to dispense could have made. The consequences have been, the loss of a valuable life; the attaching of undeserved censure to one of the surgeons of the institution at which the mistake occurred; the dragging of this gentleman, and another meritorious medical officer before a public tribunal, in a most improper manner, and subjecting them to treatment, as harsh as it was uncalled for ; and, lastly, the bringing of the institution itself into discredit and disrepute.

These are mischiefs which the provision of a sufficient amount of competent assistance would have avoided; for nothing tends more to injure the efficiency of any institution, of whatever description, than accunulating on one individual the duties of others. If the apothecary of an Infirmary or Dispensary is to be called upon to prescribe in the absence of the authorized medical officer, physician, or surgeon, as it may be, his place in the dispensing department of the institution for the time being must be otherwise filled up. It becomes the duty therefore of the Board of Management to see that the assistant who is to take his place be properly qualified, - a person who has had a sufficient experience in the compounding of medicines, who knows at least their names and general properties, and who has a competent knowledge of the language and terms in which prescriptions are written.

Now, co mere porter, however useful such an underling may be in waiting upon the dispenser, 